Washington University School of Medicine Digital Commons@Becker

\title{
Additive global cerebral blood flow normalization in arterial spin labeling perfusion imaging
}

\author{
Stephanie B. Stewart \\ Washington University School of Medicine in St. Louis \\ Jonathan M. Koller \\ Washington University School of Medicine in St. Louis \\ Meghan C. Campbell \\ Washington University School of Medicine in St. Louis \\ Joel S. Perlmutter \\ Washington University School of Medicine in St. Louis \\ Kevin J. Black \\ Washington University School of Medicine in St. Louis
}

Follow this and additional works at: https://digitalcommons.wustl.edu/open_access_pubs

Please let us know how this document benefits you.

\section{Recommended Citation}

Stewart, Stephanie B.; Koller, Jonathan M.; Campbell, Meghan C.; Perlmutter, Joel S.; and Black, Kevin J., "Additive global cerebral blood flow normalization in arterial spin labeling perfusion imaging." PeerJ. 3 , e834. (2015).

https://digitalcommons.wustl.edu/open_access_pubs/3778

This Open Access Publication is brought to you for free and open access by Digital Commons@Becker. It has been accepted for inclusion in Open Access Publications by an authorized administrator of Digital Commons@Becker. For more information, please contact vanam@wustl.edu. 


\title{
PeerJ
}

\section{Additive global cerebral blood flow normalization in arterial spin labeling perfusion imaging}

\author{
Stephanie B. Stewart ${ }^{1,2}$, Jonathan M. Koller ${ }^{2}$, Meghan C. Campbell ${ }^{1,3}$, \\ Joel S. Perlmutter ${ }^{1,3,4,5,6}$ and Kevin J. Black ${ }^{1,2,3,4,5}$ \\ ${ }^{1}$ Department of Neurology, Washington University School of Medicine, St Louis, MO, USA \\ 2 Department of Psychiatry, Washington University School of Medicine, St Louis, MO, USA \\ ${ }^{3}$ Department of Radiology, Washington University School of Medicine, St Louis, MO, USA \\ ${ }^{4}$ Departments of Anatomy and Neurobiology, Washington University School of Medicine, St \\ Louis, MO, USA \\ ${ }^{5}$ Division of Biology and Biomedical Sciences, Washington University School of Medicine, St \\ Louis, MO, USA \\ ${ }^{6}$ Programs in Physical Therapy and Occupational Therapy, Washington University School of \\ Medicine, St Louis, MO, USA
}

\section{ABSTRACT}

To determine how different methods of normalizing for global cerebral blood flow (gCBF) affect image quality and sensitivity to cortical activation, pulsed arterial spin labeling (pASL) scans obtained during a visual task were normalized by either additive or multiplicative normalization of modal gCBF. Normalization by either method increased the statistical significance of cortical activation by a visual stimulus. However, image quality was superior with additive normalization, whether judged by intensity histograms or by reduced variability within gray and white matter.

Submitted 31 December 2014 Accepted 20 February 2015 Published 17 March 2015

Corresponding author Kevin J. Black, kevin@WUSTL.edu

Academic editor

Darren Gitelman

Additional Information and Declarations can be found on page 10

DOI 10.7717/peerj.834

Copyright

2015 Stewart et al.

Distributed under Creative Commons CC-BY 4.0

\section{OPEN ACCESS}

Subjects Neuroscience, Neurology

Keywords Cerebral blood flow, ASL, Arterial spin labeling, fMRI,

Functional magnetic resonance imaging, Cerebral blood flow measurement, Parkinson's disease

\section{INTRODUCTION}

Blood flow imaging of the brain has brought insights to neuroscience for over 50 years (Taber, Black \& Hurley, 2005). Whole-brain, or global, cerebral blood flow (gCBF) calculated from blood flow images can be the outcome measure of interest, or can permit correction for gCBF fluctuations that complicate identifying or interpreting relative changes in regional blood flow (rCBF) (Black et al., 2002).

Readers more familiar with BOLD (blood oxygen level dependent) imaging must take care not to confuse (a) gCBF calculation and correction with (b) correction for global signal in statistical analysis of BOLD images, a procedure that differs in several important ways. Unlike typical BOLD methods, CBF is a physical attribute with physiologically meaningful values. The typical distribution of CBF across the healthy human brain is well established by a variety of techniques, with a mean of about $50 \mathrm{ml}$ of blood per $100 \mathrm{~g}$ of brain tissue per minute $(50 \mathrm{ml} / \mathrm{hg} / \mathrm{min}$; solid curve in Fig. 1) (Lassen, 1985; Ramsay et al., 1993). Therefore, any CBF imaging method can be verified by quantitative comparison to 


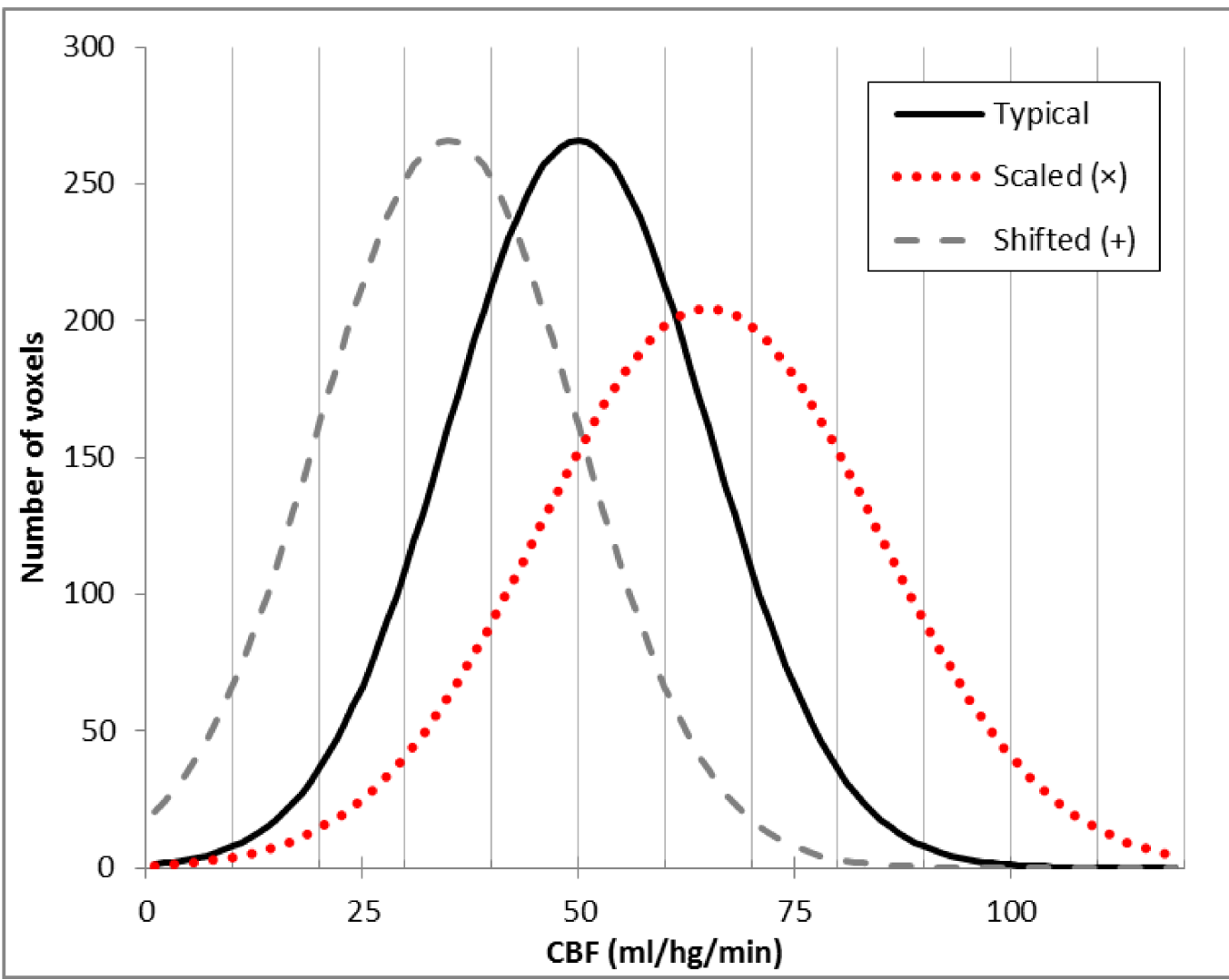

Figure 1 Typical brain rCBF values and the effects of multiplication or addition. Histogram of typical rCBF values from resting human brain (solid line). Histograms are also shown for the voxelwise product of that image with 1.3 (dotted line) and for the difference image after subtracting $20 \mathrm{ml} / \mathrm{hg} / \mathrm{min}$ from each voxel (dashed line).

these standard values. As a consequence, voxels within a single CBF image are expected to demonstrate internally consistent values (e.g., gray matter $\mathrm{CBF}$ is similar throughout the brain), again unlike most BOLD methods. Determining global CBF is thus a method aimed primarily at calibrating the voxels within a single CBF image, not a method for accounting for fluctuations at a single voxel across a time series of BOLD images. Another way of saying this is that gCBF computation is aimed at finding a numerical value with physical units, rather than the value of a test statistic.

Early in vivo measures of CBF in humans used PET to collect an autoradiographic image of radioactive counts over a defined interval of time (Herscovitch, Markham \& Raichle, 1983). Measuring arterial radioactivity over time allowed calculation of quantitative $\mathrm{rCBF}$ from the PET image of radioactivity concentration (Herscovitch, Markham \& Raichle, 1983; Raichle et al., 1983). Changes in rCBF then could be calculated directly by subtracting two different PET images of rCBF (Ramsay et al., 1993). However, arterial sampling required an invasive measurement and added statistical noise. Fortunately, the quantitative CBF image was essentially a scalar multiple of the PET radioactivity concentration image, so when gCBF was not expected to change substantially between two CBF images, one could 
multiplicatively scale the raw images to an arbitrary gCBF value before subtraction, and many PET activation studies did just that to account for modest fluctuations of gCBF between PET scans without arterial sampling for quantification (Fox et al., 1984).

In 2008 we performed an ASL pharmacological challenge MRI study in Parkinson disease (PD) (Black et al., 2010b). The original analysis used multiplicatively scaled images based on our experience with PET blood flow imaging (Black et al., 2002). However, when we revisited those data recently for a new analysis (Stewart et al., 2014), we sought to improve the quality of the scaled images. Frequency histograms of the perfusion images appeared to differ from the typical rCBF distribution by a fixed shift (dashed line in Fig. 1) rather than by a similar change in mean and dispersion, as one would see with multiplicatively scaled images (dotted line in Fig. 1). Returning to basic principles suggested an explanation. Unlike PET CBF, perfusion images from arterial spin labeling (ASL) MRI are created by subtracting two images obtained a few seconds apart, one in which arterial blood flowing into the brain has been labeled using a spatially limited radio frequency pulse ("tag") and a second image without that label ("control") (Wang et al., 2003). Subtraction creates the possibility of a negative or positive additive bias across a CBF image, in which case additive rather than multiplicative correction may better equalize the intensity of two ASL CBF images.

We test that hypothesis here using several criteria to assess image quality.

\section{MATERIAL \& METHODS}

\section{Study participants}

Twenty-one nondemented, nondepressed, ambulatory adults age 40-75 with idiopathic $\mathrm{PD}$, treated with a stable dose of levodopa but no dopamine agonists, participated in the study. Detailed inclusion and exclusion criteria and subject characteristics were reported previously (Black et al., 2010a; Black et al., 2010b). Subjects were enrolled in a Phase 2a dose-finding study (Black et al., 2010b), but here we use only data acquired on the placebo day when subjects were in the "practical off state" (i.e., no antiparkinsonian medications for at least $9 \mathrm{~h}$ ). The study was approved by the Washington University Human Research Protection Office (IRB approval \# 08-0059), and all subjects provided written documentation of informed consent prior to participation.

\section{Subject behavior}

Each subject had two perfusion MRI scans while fixating a central crosshair surrounded by a circular checkerboard reversing at $8 \mathrm{~Hz}$, and two control visual fixation scans with the crosshair but no checkerboard.

\section{MR image acquisition}

All MRI data were acquired on a Siemens 3T Tim Trio with matrix head coil. ASL images were acquired with the commercial Siemens pulsed arterial spin labeling (pASL) sequence (Wang et al., 2003). Fifteen transverse echo-planar readout slices were acquired with center-to-center slice distance $7.5 \mathrm{~mm}, 64 \times 64$ voxels in plane with dimensions $(3.4375 \mathrm{~mm})^{2}$, repetition time (TR) $2.6 \mathrm{~s}$, echo time (TE) $13.0 \mathrm{~ms}$, and flip angle $90^{\circ}$. An 
$\mathrm{M}_{0}$ image was followed by 31 tag-control pairs for a total acquisition time for each ASL "scan" of $2.73 \mathrm{~min}$.

Brain structure was assessed from sagittal MP-RAGE acquisitions with voxel size $(1.0 \mathrm{~mm})^{3}, \mathrm{TR}=2.4 \mathrm{~s}, \mathrm{TE}=3.08 \mathrm{~ms}, \mathrm{TI}=1,000 \mathrm{~ms}$, flip angle $=8^{\circ}$. The structural images for each subject were inspected visually, images of lower quality were rejected, and the remaining 1-4 MP-RAGE images for each subject were mutually registered.

\section{Image registration and creation of CBF images}

The 63 frames of the ASL run were rigidly aligned using a validated method (Black et al., 2001) and summed to facilitate later alignment steps. Each frame was smoothed using a Gaussian filter with $7.35 \mathrm{~mm}$ kernel (FWHM), and cerebral blood flow (CBF) was computed in each voxel for each tag-control pair as described (Wang et al., 2003). The summed, aligned EPI images from each run were mutually aligned within each subject and summed across runs, and the resulting image was affine registered to a target image in Talairach and Tournoux space made using validated methods from the structural MR images from these subjects (Black et al., 2004). The products of the registration matrix from this step and the matrices from the within-run mutual registration step were used to resample the 31 tag-control pair CBF images from each run into atlas space in a single resampling step. A whole-brain binary image was created from the atlas-space structural target image, and all analyses below included only the CBF image voxels corresponding to voxels within this mask image.

To minimize motion-related artifact we removed tag-control pairs from all further analyses if framewise displacement in either EPI image exceeded $0.9 \mathrm{~mm}$ as defined by Siegel et al. (2014). The remaining CBF images in atlas space were averaged to create a single atlas-registered CBF image for each ASL run. One subject's data was excluded from further analysis because over half of the frame pairs were removed due to head motion.

\section{Image intensity correction}

\section{Estimating modal CBF image intensity}

We evaluated each CBF image independently for each of the 4 scans in each subject. The image intensity histograms were constructed with bins 1 unit wide, so were not smooth. We chose to normalize image intensity based on the idealized peak of this distribution (which if there were no noise would be the mode, i.e., the most common voxel intensity in the brain) (Ojemann et al., 1997). Specifically, the method of least squares was used to identify the vertex of the parabola that best fit the histogram using voxels within $70 \%$ of the mode bin (Fig. 2).

\section{Additive and multiplicative intensity correction}

Each input image was corrected in two ways: multiplicatively (multiplying every voxel in the image by 50/mode), and additively (adding 50-mode to every voxel), so that the idealized modal CBF for every corrected image was 50 (nominal units $\mathrm{mL} / \mathrm{hg} / \mathrm{min}$ ). 


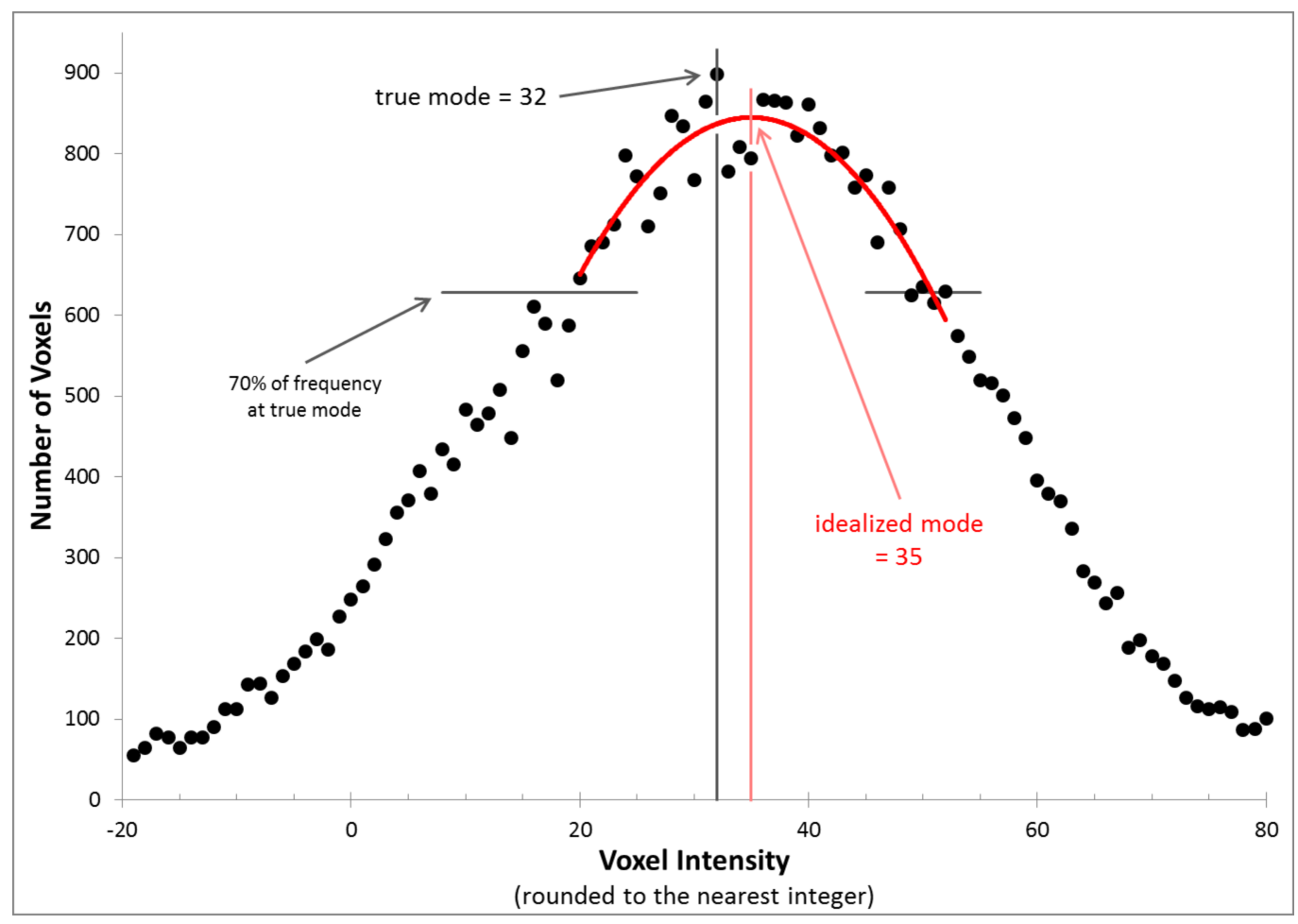

Figure 2 Determining the idealized mode. The figure shows the frequency histogram for one of the CBF images. With this bin width, the true mode falls at 32 (frequency $=898$, bin including all voxels with values 31.5-32.5), but the mean of the distribution and the vertex of the parabola shown (the idealized mode) both fall at 35.0 .

\section{Defining volumes of interest}

Gray matter (GM), white matter (WM) and visual cortex volumes of interest (VOIs), excluding CSF regions, were defined from each subject's high-resolution MP-RAGE image by FreeSurfer (version 5.3, http://surfer.nmr.mgh.harvard.edu) (Desikan et al., 2006). VOIs were limited to voxels that were represented in every image in every subject; this step excluded much of the inferior occipital cortex in the visual cortex VOI (Black et al., 2010b). We also used the thalamic VOIs previously defined in our drug study (Black et al., 2010b).

\section{Statistical analysis}

To determine the effect of gCBF normalization on task effect, all statistical analyses were performed in triplicate, one for each set of images: uncorrected (before removing the gCBF effect), multiplicatively normalized, and additively corrected.

\section{Image quality analysis}

Using only the fixation scans, the mean $\mathrm{rCBF}$ and its variability in the GM, thalamic and WM VOIs was determined. The gray to white matter CBF ratio was also calculated. 


\section{VOI analysis}

To determine the change in $\mathrm{rCBF}$ in a VOI with visual stimulation, the mean CBF across all voxels in the VOI from the fixation scans was subtracted from the corresponding mean in the task scans. Statistical significance was assessed with paired $t$ tests.

\section{Statistical images}

To identify regions activated by the visual task, we used a mixed-effects approach. First, for each study subject, changes in rCBF were identified using SPM12b software (www.fil.ion. ucl.ac.uk/spm/) and a voxelwise general linear model that included task (checkerboard) versus control (fixation). The task contrast was used to generate an image of the weighted parameter estimate for each subject. These single-subject contrast images were used as input for a second SPM analysis using a voxelwise general linear model that included a covariate for subject age and a factor for sex. Statistical inference was performed at each voxel with a one-sample $t$ test (i.e., testing whether the task contrast images are significantly less than or greater than zero, across subjects). After thresholding at $p=0.001$, multiple comparisons correction was performed with the cluster false discovery rate set at $p=0.05$. Approximate anatomical locations of peaks in the statistical images were provided by the Talairach Daemon client (www.talairach.org) (Lancaster et al., 2000).

\section{RESULTS}

We assessed the effect of additive or multiplicative intensity equalization in several ways: by examining the voxel intensity distribution (by frequency histogram), by judging image quality visually, by the variability of voxel intensity within the GM and WM, and by the suitability of each set of images for detecting appropriate brain activation with visual stimulation.

\section{Voxel intensity distribution (histogram)}

The idealized mode \pm SD for the original CBF images was $33.36 \pm 7.30$. The original $\mathrm{CBF}$ images contained a reasonable distribution of voxel intensities except that many of them appeared shifted leftwards to varying degrees, so that many voxels in the brain had physiologically impossible negative values (the 3 curves in the histogram in Fig. $3 \mathrm{~A}$ reflect all brain voxels from 3 successive ASL images from the same subject; a transverse section from each of these images is shown to the right of the histogram). Multiplicative normalization of course produced an image with an equal fraction of negative voxel values, though the normalized image's mode was now 50 (histograms in Fig. 3B). Additive normalization produced a voxel intensity distribution that reflects the physiological expectation. In addition, it much more accurately matches the intensity distribution widths across the normalized images (histograms in Fig. 3C).

\section{Image quality}

Images normalized additively (Fig. 3C) appear to show more uniform voxels within the GM and WM. To corroborate this quantitatively, we determined the variability in the GM and WM both between subjects and within subjects, using scans from the fixation task only. 


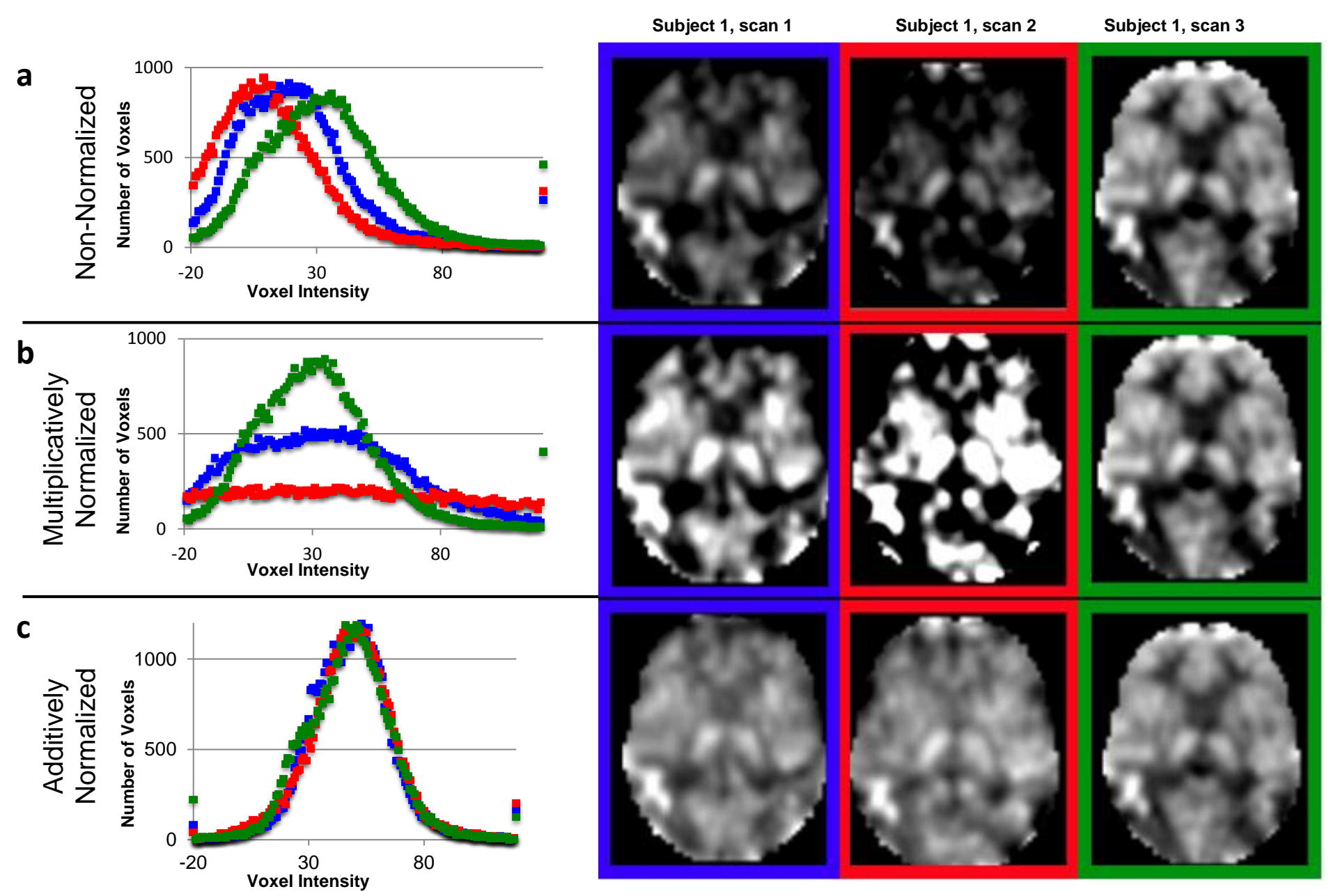

Figure 3 Additive intensity normalization improves pASL image quality. The effect of multiplicative or additive intensity normalization on 3 ASL scans (represented by red, blue and green) from one single subject: (A) before normalization, (B) after multiplicative normalization, (C) after additive normalization. The histograms show the frequency of the voxel values across the brain (i.e., within an anatomically-defined brain mask only) for each of 3 sequential scans. The same transverse slice in atlas space from the 3 sequential scans is also shown; scan 1 (blue), scan 2 (red) and scan 3 (green). The grayscale range is set at $0-100 \mathrm{ml} / \mathrm{hg} / \mathrm{min}$, a range that reasonably reflects true physiological values, except for the images before normalization (A), for which the range is set at $0-75 \mathrm{ml} / \mathrm{hg} / \mathrm{min}$ because otherwise the structure in some of the images would be obscured.

Across subjects, the mean GM intensities are more similar in additively normalized scans (coefficient of variation $[\mathrm{CV}]=0.145$ ) as compared to non-normalized (CV $=0.194)$ or multiplicatively normalized scans $(\mathrm{CV}=0.266)$. Within the $\mathrm{GM}$, the thalamic VOI also had mean intensities more similar in additively normalized scans (coefficient of variation $[\mathrm{CV}]=0.0093)$ as compared to non-normalized $(\mathrm{CV}=0.154)$ or multiplicatively normalized scans $(\mathrm{CV}=0.167)$. The WM intensities are more similar in additively normalized scans $(\mathrm{CV}=0.158$; non-normalized $\mathrm{CV}=0.206$, multiplicatively normalized scans $\mathrm{CV}=0.263)$. The GM:WM ratio is more similar across subjects after additive normalization $(\mathrm{CV}=0.056$; non-normalized $\mathrm{CV}=0.074$, multiplicatively normalized $C V=0.072)$ (Table 1$)$. 
Table 1 Variability across subjects of mean GM, thalamus and WM rCBF.

\begin{tabular}{|c|c|c|c|}
\hline & $\begin{array}{l}\text { Non-normalized } \\
\text { images }\end{array}$ & $\begin{array}{l}\text { Multiplicatively } \\
\text { normalized }\end{array}$ & $\begin{array}{l}\text { Additively } \\
\text { normalized }\end{array}$ \\
\hline Mean GM rCBF $(S D, C V)^{a}$ & $38.78(7.51,0.194)$ & $62.02(16.47,0.266)$ & $55.89(8.11,0.145)$ \\
\hline Mean Thalamus rCBF (SD, CV) & $54.80(8.42,0.154)$ & $84.66(14.10,0.167)$ & $71.41(6.67,0.093)$ \\
\hline Mean WM rCBF (SD, CV) & $27.56(5.68,0.206)$ & $43.85(11.54,0.263)$ & $44.67(7.06,0.159)$ \\
\hline Mean GM:WM ratio (SD, CV) & $1.416(0.104,0.074)$ & $1.418(0.102,0.072)$ & $1.256(0.070,0.056)$ \\
\hline
\end{tabular}

Table 2 rCBF changes in visual cortex and WM VOIs with visual stimulation.

\begin{tabular}{llll} 
& $\begin{array}{l}\text { Non-normalized } \\
\text { images }\end{array}$ & $\begin{array}{l}\text { Multiplicatively } \\
\text { normalized }\end{array}$ & $\begin{array}{l}\text { Additively } \\
\text { normalized }\end{array}$ \\
\hline $\begin{array}{l}\text { Mean change in rCBF (SD, CV) in } \\
\text { visual cortex region }\end{array}$ & $17.70(13.88,0.784)$ & $29.83(18.91,0.634)$ & $18.15(13.37,0.737)$ \\
$p$ & $1.70 \times 10^{-5}$ & $1.03 \times 10^{-6}$ & $7.72 \times 10^{-6}$ \\
$\begin{array}{l}\text { Mean change in rCBF (SD, CV) } \\
\text { in white matter region }\end{array}$ & $1.07(3.23,3.01)$ & $2.85(5.99,2.10)$ & $1.53(3.47,2.28)$ \\
$p$ & 0.15 & 0.05 & 0.06 \\
\hline
\end{tabular}

Notes.

${ }^{a}$ Nominal units $\mathrm{ml} / \mathrm{hg} / \mathrm{min}$. Note that the mean changes are not strictly comparable between methods because of the multiplication. Hence we provide SD and $p$ values for comparison.

\section{Task activation: a priori volume of interest}

We examined the effect of visual stimulation in the partial visual cortex VOI (intended as a positive control) and in the WM VOI (intended as a negative control). With this substantial visual stimulus, the signal is detected even without normalization (visual cortex VOI, $p=1.70 \times 10^{-5}$, paired $t$ test), but the statistical significance of the change is greater in either set of normalized images (multiplicative normalization, $p=1.03 \times 10^{-6}$, additive normalization, $p=7.72 \times 10^{-6}$ ). The mean change in $\mathrm{rCBF}$ in the visual cortex VOI increased when the images were multiplied, but was always $>10$ times higher than the change in the WM control VOI (Table 2).

\section{Task activation: statistical image}

Similarly to the VOI analysis, with this substantial visual stimulus, SPM analyses of visual stimulation identified significant occipital cortex activation even without normalization, though normalization increased the peak $t$ and the volume of significant clusters (Fig. 4 and Table 3). For all 3 analyses, the peak $t$ value occurred in the occipital lobe, in Brodmann area 17 or 18 . There were no significant clusters of deactivation with any of the analyses.

\section{DISCUSSION}

Additive normalization of gCBF is superior to multiplicative normalization for this pASL technique, judged by image intensity distributions and by homogeneity within gray matter 


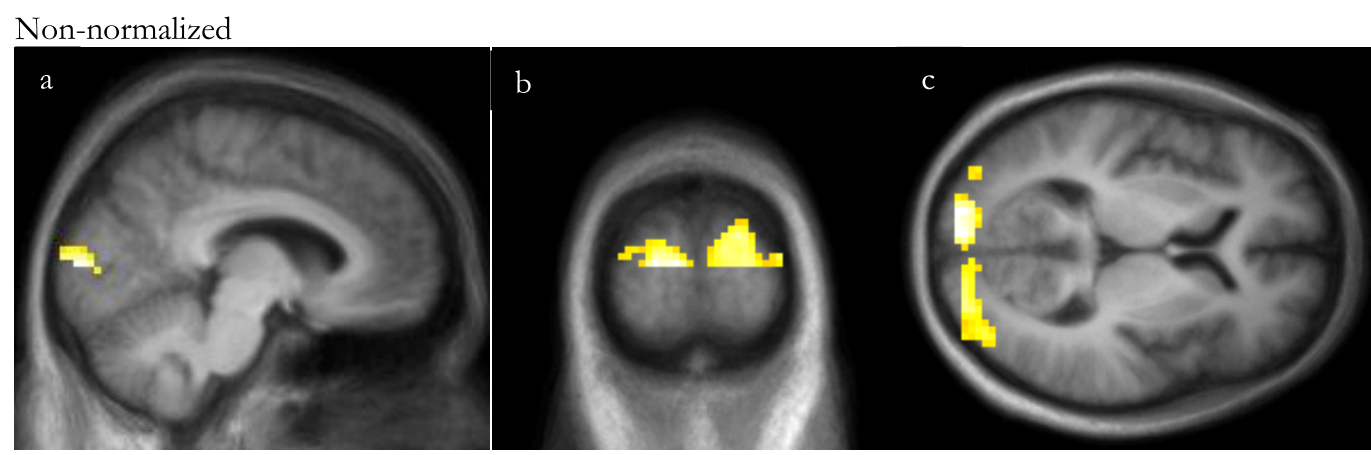

Multiplicatively Normalized

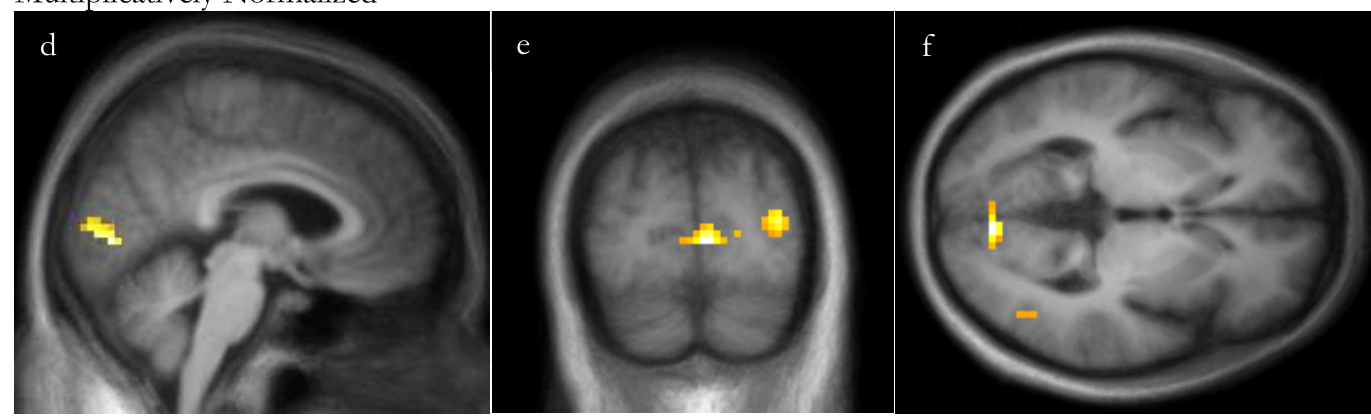

Additively Normalized

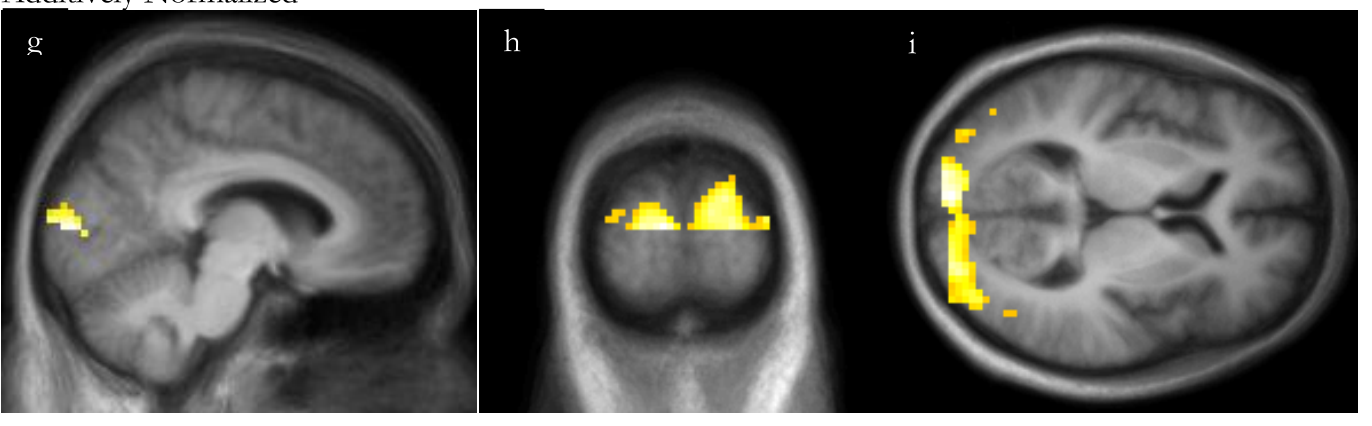

Figure 4 SPM regions of activation. Top row: before normalization with (A) sagittal, (B) coronal and (C) axial slices; middle row: after multiplicative normalization (D) sagittal, (E) coronal and (F) axial slices; bottom row: after additive normalization with $(\mathrm{G})$ sagittal, $(\mathrm{H})$ coronal and $(\mathrm{I})$ axial slices. The lower half of occipital cortex was excluded from this analysis due to incomplete superior-inferior coverage in some subjects (see Methods: Defining volumes of interest).

and white matter. Multiplicative scaling increases the variability in GM and WM, whereas additive normalization improves image quality and reduces variability between subjects.

We previously found that correcting for gCBF improved task signal in ASL in young, healthy volunteers (Black et al., 2008; Henniger et al., 2009). The present report extends those findings to patients with Parkinson disease. Normalizing scans for variability in gCBF improves detection of statistically significant activations from a visual task, whether analyzed using an anatomically-defined visual cortex VOI or by whole-brain SPM. Although noise properties improve to a greater extent with additive scaling, either 
Table 3 Activation by a visual stimulus: SPM analyses.

\begin{tabular}{llll} 
& $\begin{array}{l}\text { Non-normalized } \\
\text { images }\end{array}$ & $\begin{array}{l}\text { Multiplicatively } \\
\text { normalized }\end{array}$ & $\begin{array}{l}\text { Additively } \\
\text { normalized }\end{array}$ \\
\hline Peak $t$ (17 d.f.) & 6.13 & 7.36 & 6.48 \\
Location of peak & $-8,-93,6$ & $4,-81,0$ & $-8,-93,6$ \\
Cluster volume, voxels $(\mathrm{ml})$ & $119(3.2)$ & $369(10.0)$ & $447(12.1)$ \\
$p$ (FDR) & $<0.0005$ & $<0.0005$ & $<0.0005$ \\
Peak $t$ for 2nd significant cluster & 5.59 & & \\
Location of peak & $26,-87,9$ & & \\
Cluster volume, voxels $(\mathrm{ml})$ & $247(6.7)$ & & \\
$p$ (FDR) & $<0.0005$ & & \\
\hline
\end{tabular}

additive or multiplicative scaling permits identification of $\mathrm{rCBF}$ responses to a strong visual stimulus.

The reader familiar with ASL should note a different and potentially confusing use of the term "global." Before calculating CBF, several pre-processing quality improvement steps are applied to the EPI images to address signal artifacts caused by head movement or MR signal drift over time within an ASL run. Wang (2012) used a general linear model and global EPI signal intensity (defined by voxels above an arbitrary intensity threshold) to account for such artifactual signal changes. We chose to address these issues using methods developed and validated at our institution; for instance, we removed motion-contaminated frames (Siegel et al., 2014) rather than modeling the consequent artifact. Again, please note that Wang's within-run global signal variable, applied separately at each voxel, is quite different from the global (whole-brain) single-scan measurement of CBF compared between runs in this communication.

\section{ADDITIONAL INFORMATION AND DECLARATIONS}

\section{Funding}

The data came from a study funded by Synosia Therapeutics. The analysis reported here was supported by NIH (K24 MH087913, T32 DA007261, NS058714, NS41509, NS075321, C06 RR020092, UL1 RR024992, P30 NS048056, U54 CA136398-02900209); the American Parkinson Disease Association (APDA) Center for Advanced PD Research at Washington University, the Greater St. Louis Chapter of the APDA, and the Barnes-Jewish Hospital Foundation (Elliot Stein Family Fund for PD Research and the Parkinson Disease Research Fund). The original study was funded commercially, but the sponsor did not participate in or affect this analysis or this report. There have been no other commercial or financial relationships that could be construed as a potential conflict of interest. The funders had no role in data analysis, decision to publish, or preparation of the manuscript. 


\section{Grant Disclosures}

The following grant information was disclosed by the authors:

Synosia Therapeutics.

NIH: K24 MH087913, T32 DA007261, NS058714, NS41509, NS075321, C06 RR020092,

UL1 RR024992, P30 NS048056, U54 CA136398-02900209.

American Parkinson Disease Association.

Center for Advanced PD Research at Washington University.

Greater St. Louis Chapter of the APDA.

Barnes-Jewish Hospital Foundation.

\section{Competing Interests}

The original study was funded commercially, but the sponsor did not participate in or affect this analysis or this report. There have been no other commercial or financial relationships that could be construed as a potential conflict of interest. Kevin Black, MD is an Academic Editor for PeerJ.

\section{Author Contributions}

- Stephanie B. Stewart analyzed the data, wrote the paper, prepared figures and/or tables.

- Jonathan M. Koller and Meghan C. Campbell conceived and designed the experiments, performed the experiments, analyzed the data, reviewed drafts of the paper.

- Joel S. Perlmutter wrote the paper.

- Kevin J. Black conceived and designed the experiments, performed the experiments, analyzed the data, wrote the paper.

\section{Human Ethics}

The following information was supplied relating to ethical approvals (i.e., approving body and any reference numbers):

Washington University Human Research Protection Office, approval \# 08-0059.

\section{Supplemental Information}

Supplemental information for this article can be found online at http://dx.doi.org/ 10.7717/peerj.834\#supplemental-information.

\section{REFERENCES}

Black KJ, Campbell MC, Dickerson W, Koller JM, Chung SC, Bandak SI. 2010a. A randomized, double-blind, placebo-controlled cross-over trial of the adenosine $2 \mathrm{a}$ antagonist SYN115 in Parkinson disease. In: Annual meeting of the American Academy of Neurology, Toronto, Canada. Minneapolis: American Academy of Neurology.

Black KJ, Duvall LB, Campbell MC, Koller JM. 2008. Signal and noise in continuous arterial spin labeling (cASL) as a function of time. In: Annual meeting, Society for Neuroscience. Washington, D.C.: Society for Neuroscience. Program \# 598.593. 
Black KJ, Hershey T, Koller JM, Videen TO, Mintun MA, Price JL, Perlmutter JS. 2002. A possible substrate for dopamine-related changes in mood and behavior: prefrontal and limbic effects of a $D_{3}$-preferring dopamine agonist. Proceedings of the National Academy of Sciences of the United States of America 99:17113-17118 DOI 10.1073/pnas.012260599.

Black KJ, Koller JM, Campbell MC, Gusnard DA, Bandak SI. 2010b. Quantification of indirect pathway inhibition by the adenosine $\mathrm{A}_{2 \mathrm{a}}$ antagonist SYN115 in Parkinson disease. Journal of Neuroscience 30:16284-16292 DOI 10.1523/JNEUROSCI.2590-10.2010.

Black KJ, Koller JM, Snyder AZ, Perlmutter JS. 2004. Atlas template images for nonhuman primate neuroimaging: Baboon and macaque. Methods in Enzymology 385:91-102 DOI 10.1016/S0076-6879(04)85006-7.

Black KJ, Snyder AZ, Koller JM, Gado MH, Perlmutter JS. 2001. Template images for nonhuman primate neuroimaging: 1. Baboon. NeuroImage 14:736-743 DOI 10.1006/nimg.2001.0752.

Desikan RS, Segonne F, Fischl B, Quinn BT, Dickerson BC, Blacker D, Buckner RL, Dale AM, Maguire RP, Hyman BT, Albert MS, Killiany RJ. 2006. An automated labeling system for subdividing the human cerebral cortex on MRI scans into gyral based regions of interest. NeuroImage 31:968-980 DOI 10.1016/j.neuroimage.2006.01.021.

Fox PT, Mintun MA, Raichle ME, Herscovitch P. 1984. A noninvasive approach to quantitative functional brain mapping with $\mathrm{H}_{2}{ }^{15} \mathrm{O}$ and positron emission tomography. Journal of Cerebral Blood Flow and Metabolism 4:329-333 DOI 10.1038/jcbfm.1984.49.

Henniger NE, Koller JM, Liou T, Matheson R, Black KJ. 2009. Quantification of the temporal stability of arterial spin labeling functional magnetic resonance imaging. In: Washington University Undergraduate Research Symposium. St. Louis: Washington University, Office of Undergraduate Research.

Herscovitch P, Markham J, Raichle M. 1983. Brain blood flow measured with intravenous $\mathrm{H}_{2}{ }^{15} \mathrm{O}$. I. Theory and error analysis. Journal of Nuclear Medicine 24:782-789.

Lancaster JL, Woldorff MG, Parsons LM, Liotti M, Freitas CS, Rainey L, Kochunov PV, Nickerson D, Mikiten SA, Fox PT. 2000. Automated Talairach atlas labels for functional brain mapping. Human Brain Mapping 10:120-131

DOI 10.1002/1097-0193(200007)10:3<120::AID-HBM30>3.0.CO;2-8.

Lassen NA. 1985. Normal average value of cerebral blood blow in younger adults is $50 \mathrm{ml} / 100$ g/min. Journal of Cerebral Blood Flow and Metabolism 5:347-349 DOI 10.1038/jcbfm.1985.48.

Ojemann JG, Akbudak E, Snyder AZ, McKinstry RC, Raichle M, Conturo TE. 1997. Anatomic localization and quantitative analysis of gradient refocused echo-planar fMRI susceptibility artifacts. NeuroImage 6:156-167 DOI 10.1006/nimg.1997.0289.

Raichle ME, Martin WR, Herscovitch P, Mintun MA, Markham J. 1983. Brain blood flow measured with intravenous $\mathrm{H}_{2}{ }^{15} \mathrm{O}$. II. Implementation and validation. Journal of Nuclear Medicine 24:790-798.

Ramsay SC, Murphy K, Shea SA, Friston KJ, Lammertsma AA, Clark JC, Adams L, Guz A, Frackowiak RS. 1993. Changes in global cerebral blood flow in humans: effect on regional cerebral blood flow during a neural activation task. Journal of Physiology 471:521-534 DOI 10.1113/jphysiol.1993.sp019913.

Siegel JS, Power JD, Dubis JW, Vogel AC, Church JA, Schlaggar BL, Petersen SE. 2014. Statistical improvements in functional magnetic resonance imaging analyses produced by censoring high-motion data points. Human Brain Mapping 35:1981-1996 DOI 10.1002/hbm.22307. 
Stewart SB, Koller JM, Campbell MC, Black KJ. 2014. Arterial spin labeling versus BOLD in direct challenge and drug-task interaction pharmacological fMRI. PeerJ 2:e687 DOI 10.7717/peerj.687.

Taber KH, Black KJ, Hurley RA. 2005. Blood flow imaging of the brain: 50 years experience. Journal of Neuropsychiatry and Clinical Neurosciences 17:441-446 DOI 10.1176/jnp.17.4.441.

Wang J, Licht DJ, Jahng GH, Liu CS, Rubin JT, Haselgrove J, Zimmerman RA, Detre JA. 2003. Pediatric perfusion imaging using pulsed arterial spin labeling. Journal of Magnetic Resonance Imaging 18:404-413 DOI 10.1002/jmri.10372.

Wang Z. 2012. Improving cerebral blood flow quantification for arterial spin labeled perfusion MRI by removing residual motion artifacts and global signal fluctuations. Magnetic Resonance Imaging 30:1409-1415 DOI 10.1016/j.mri.2012.05.004. 